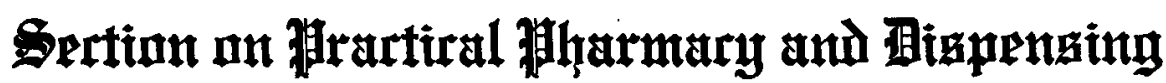

\author{
Papers Presented at the Sixty-Second Annual Convention
}

\section{ASSAYING GALENICALS.}

JEANNOT HOSTMANN.

At the Nashville meeting, the writer contributed a paper on "The Necessary Apparatus in a Retail Pharmacy," which did not create much discussion. Since then, it has been both praised and criticized. Very little praise emanated from retail pharmacists but quite some criticism. The praise was usually bestowed by men not actively engaged in the retail business.

At the annual meeting of one of the state associations, the writer heard a member criticize the enforcement of the "Food and Drug Law" or rather laws, and was surprised when the statement was made that if retail pharmacists attempted to assay and standardize their drugs and preparations, the cost would be prohibitive.

"Are we druggists supposed to spend a thousand dollars for the equipment of an analytical laboratory, and then spend twelve hundred or more dollars per year to hire a chemist to run it for us?" This was his query. He did not, as I do not believe any progressive pharmacist would, deny that assaying and standardizing were absolutely necessary to the welfare of our profession; he simply, like many others, seemed to think it more important to attempt to standardize the price of "Brown's Cureall" than to raise pharmacy to a professional level in the eyes of the physician and layman by actually assuring himself by tests made in his establishment that the drugs he was selling and dispensing were really as represented.

Several papers have been read at various meetings during the current year, discussing the separation of the professional pharmacy from the commercial drug store. A paper will be read at this meeting in which the author recommends the formation of a national association of prescriptionists. All this shows the trend of thought of many thinking pharmacists, which no doubt will sooner or later bear fruit in placing pharmacy where it belongs, viz: A profession, whose first thought will always be to save human life and alleviate and prevent human suffering.

Màrtin I. Wilbert, in Reprint No. 189 from the Public Health Reports, after showing a summary of adulterated and sophisticated drugs collected during the time of one year, calls attention to the duty of pharmacy as follows:

"In conclusion it may be reiterated that the more evident shortcoming in the present-day enforcement of pure-drugs laws is the general failure to properly place the responsibility for the nature, kind and purity of the medicines supplied

'J. A. Ph. A., 1914, 697. 
to the consumer where it belongs. This shortcoming is being corrected, to some extent at least, by recently enacted laws to regulate the practice of pharmacy by placing the responsibility squarely on the person dispensing the drug.

"The proper enforcement of laws designed to regulate the practice of pharmacy in conjunction with pure-drugs laws, should relieve physicians and the public of any doubt as to the composition, purity, quality and strength of all drugs and medicinal preparations used in the treatment of disease. As these laws are enforced at the present time it is plainly evident that the methods of control are inadequate and do not serve to safeguard public health as well as they could or should.

"Boards of health and other State and Federal officials intrusted with the enforcement of these laws should endeavor to call attention to the desirability of having druggists exercise a close scrutiny of the drugs and preparations included in their stock, to keep drugs, chemicals and preparations in suitable containers. to throw away old useless material, and to see that scales, weights, and measures are reliable and accurate under the conditions imposed upon them.

"Some effort should also be made to see that drug stores are equipped with the necessary analytical apparatus with which to analyze or examine all supplies and thus assist in maintaining a more efficient control of the articles sold as medicine.

"Consistent and efficient control of the identity, purity and strength of all drugs and preparations as furnished the consumer would make for progress in the science of medicine and should prove to be an important factor in promoting public health."

The United States Pharmacopoia presents the tests in such a way that any pharmacist who is a graduate of any good college of pharmacy and cares to employ the knowledge that he has gained as a student will have no trouble in determining whether the drugs and chemicals.he purchases and dispenses and the preparations thereof, are up to standard.

If the pharmacist is a graduate of a college of pharmacy where pharmacy is taught as it should be, and employs only such graduates in his Prescription Department, he will not have to hire any chemist to attend to this class of work although it may be necessary for him or his employee to spend some of the time now spent in dressing windows, etc., in the laboratory.

The false ideas as to cost of equipment caused the writer to compile the following table which embraces the most important utensils needed to perform the necessary tests:

INVENTORY OF EQUIPMENT AND COST.

Volumetric Flasks.

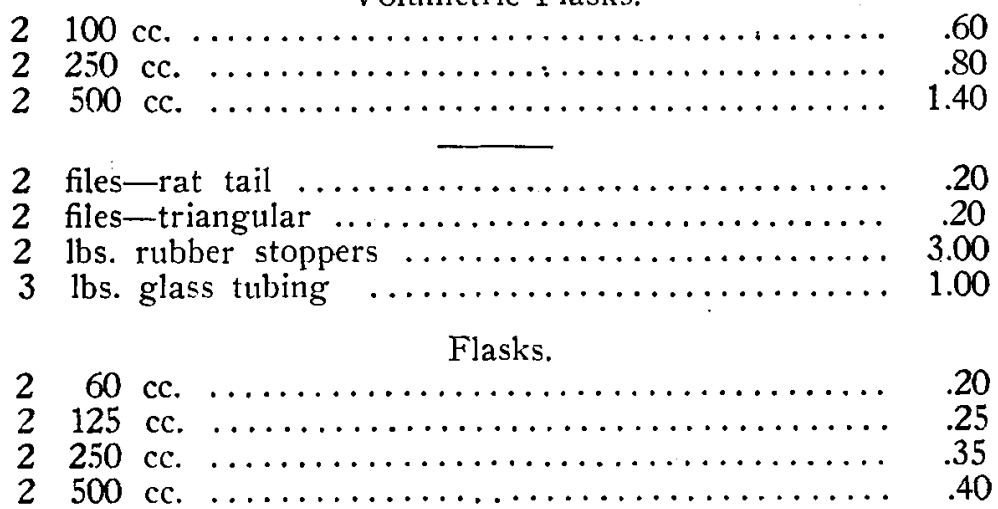




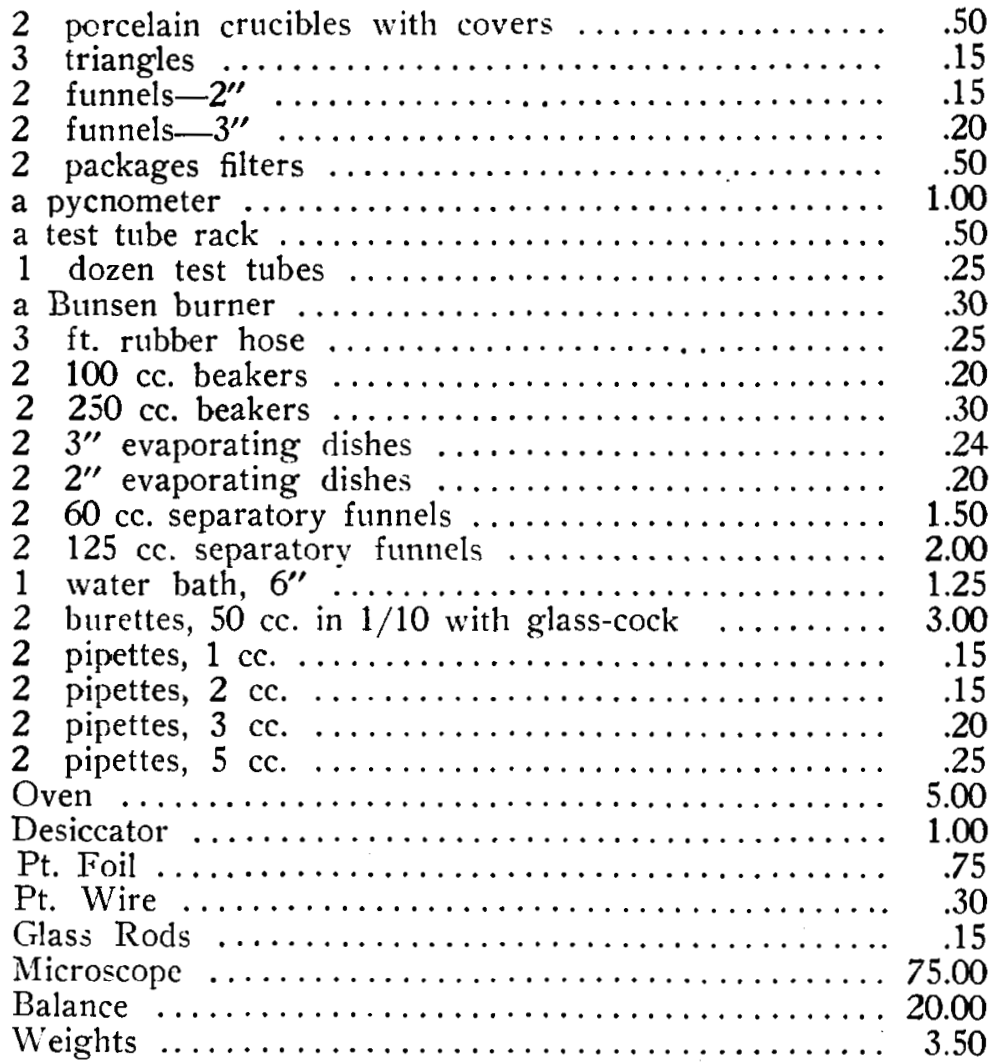

Thus you will see it will cost about twenty-five dollars to buy the most necessary utensils, if the store is not equipped with a good balance, and many are not, why then twenty-five dollars more will buy the balance and weights from one milligram to one hundred grams. An oven and desiccator will cost six dollars more and, finally, a good microscope can be purchased for seventy-five dollars.

This comparatively small outlay will repay the pharmacist, who intelligently and faithfully uses the outfit after installation, many times over financially, as woll as otherwise.

Columbia University, College of Pharmacy.

The value of the exports of perfumery to the United States from the consular district of Nice, France, decreased from $\$ 1,578,228$ for 1913 to $\$ 755,671$ for last year. 